\title{
Metronomic chemotherapy for elderly and/or frail patients
}

The results of a phase II trial led by

Hans Wildiers show that, for older patients with metastatic HER2-positive disease, the addition of metronomic cyclophosphamide to the combination trastuzumab plus pertuzumab (TP) leads to markedly longer progression-free survival (PFS) durations compared with TP alone.

The investigators chose metronomic chemotherapy because of its lower risk of toxicities compared with docetaxel, which is usually administered in combination with TP. “The goals of treatment become different with increasing age: overall survival is less relevant in older women than in younger patients. Avoidance of classical chemotherapy is an important goal for the elderly," Wildiers explains.

Patients in this study had chemotherapynaive metastatic HER2-positive breast cancer and were either at least 70 years old, or $\geq 60$ years old and functionally impaired as defined by a series of predefined criteria. Participants were randomly assigned to receive
TP alone $(n=39)$ or supplemented with metronomic oral cyclophosphamide (TPM; $n=41$ ). At 20.7 months, patients receiving TPM had longer PFS durations than those receiving TP (12.7 months versus 5.6 months).

Patients with disease progression during treatment were eligible for subsequent monotherapy with trastuzumab entamsine. This treatment, which was given to 29 patients in this study, resulted in median disease-control durations of 5 months.

Of note, all of the regimens tested had acceptable toxicity profiles. "The benefit of avoiding the toxicity of chemotherapy by administering TP only does not compensate for a substantial loss of activity. Antitumour activity remains higher when a 'gentle' chemotherapy is added to TP," Wildiers concludes.

Diana Romero

ORIGINAL ARTICLE Wildiers, H. et al. Pertuzumab and

trastuzumab with or without metronomic chemotherapy for older patients with HER2-positive metastatic breast cancer (EORTC 75111-10114): an open-label, randomised, phase 2 trial

from the Elderly Task Force/Breast Cancer Group. Lancet Oncol. https://doi.org/ 10.1016/S1470-2045(18)30083-4 (2018) 